A fast method to measure the irradiance response of image processing systems

This article has been downloaded from IOPscience. Please scroll down to see the full text article.

1995 Meas. Sci. Technol. 6181

(http://iopscience.iop.org/0957-0233/6/2/009)

View the table of contents for this issue, or go to the journal homepage for more

Download details:

IP Address: 147.96.14.16

The article was downloaded on 14/05/2013 at 19:17

Please note that terms and conditions apply. 


\title{
A fast method to measure the irradiance response of image processing systems
}

\author{
J A Quiroga†, A González-Canoł and E Bernabeu† \\ †Departamento de Óptica, Universidad Complutense de Madrid, Facultad de Ciencias \\ Físicas, Ciudad Universitaria s/n, 28040 Madrid, Spain \\ ‡Departamento de Óptica, Escuela Universitaria de Óptica, Universidad Complutense, \\ Arcos de Jalón s/n, 28037 Madrid, Spain
}

Received 18 July, in final form 14 September 1994 , accepted for publication 27 October 1994

\begin{abstract}
A method to estimate in a fast and simple way the response of an image acquiring system (such as a charge-coupled device camera plus an image-processing board) for a monochromatic light source is presented. Two images, whose irradiances are related in a known way, are acquired, and the corresponding grey levels of the responses of the system are correlated pixel to pixel. From this correlation and the relationship between the irradiances of the images, a relative estimation of the irradiance response of the system can be obtained by means of simple algorithms. This method is fast and easy to implement and the results obtained are satisfactory.
\end{abstract}

\section{Introduction}

Image processing systems based on $\mathrm{CCD}$ cameras and processing boards are used in a great number of fields. For certain applications, such as laser beam characterization [1] and phase-shifting interferometry [2], a good knowledge of the irradiance response of the CCD plus board system is required, so that errors due to nonlinearities in the response can be corrected, using the input 'look-up' tables (LUTS) of the processing board. Although CCD imagers are inherently linear, the use of the imageprocessing board may introduce nonlinearities [3,4]. For instance, Creath refers to an error of 0.02 waves in the phase calculated with a 'three buckets' algorithm for a second-order nonlinear detection error of $10 \%$, as figure 17 of [2] shows. On the other hand, for a Gaussian beam a second-order nonlinear detection error of $10 \%$ produces an error of the order of $7 \%$ in the estimation of the second moment (width) of the laser beam.

The irradiance response of an image-acquiring system is usually measured by taking a series of uniform images of known irradiances. Then, the average grey level of the response is represented as a function of irradiance [3].

In this paper, we present a method to estimate the relative irradiance response of a $\mathrm{CCD}$ plus processing board system that reduces the measuring time. Only two images are needed and the algorithms involved are very simple and easy to implement. The method is fast enough to allow system response monitoring as the processing system warms up. The absolute irradiance response can be obtained when the response of the system for only one value of the irradiance is known (this datum is usually provided by the manufacturer).

\section{The theoretical basis}

Let us consider the irradiance distributions produced by two images on the image plane of a CCD array, $E_{1}(x, y)$ and $E_{2}(x, y)$. Let us suppose that between them there exists a relationship, namely,

$$
E_{1}(x, y)=f\left(E_{2}(x, y)\right)
$$

where $f$ is a function only of irradiance, independent of position.

We will characterize the irradiance response of the image-processing system by a function $T(E)$, where $T(E)$ denotes the output grey level given by the system for an input irradiance $E$. As with $f$, we suppose that $T$ does not depend on position. $T$ will have a range of values imposed by the number of bits employed in the digitization of the image by the system.

In this way, the system outputs to the incident irradiance inputs are

$$
\begin{aligned}
& I_{1}(x, y)=T\left(E_{1}(x, y)\right) \\
& I_{2}(x, y)=T\left(E_{2}(x, y)\right)
\end{aligned}
$$

We need to establish a relationship between $I_{1}$ and $I_{2}$ in a grey level basis. To do so, we can consider for every point the output grey level for each incident irradiance distribution (let us call them $G_{1}$ and $G_{2}$ for simplicity; of course, $G_{i}=I_{i}(x, y), i=1,2$; and we represent on a coordinate system the pairs of grey levels for all the points (figure 1).

In an ideal case, a grey level $G_{1}$ will be transformed to a grey level $G_{2}$ independently of the point where these grey levels have been measured. Owing to problems of noise or inaccuracies during the acquisition of the 


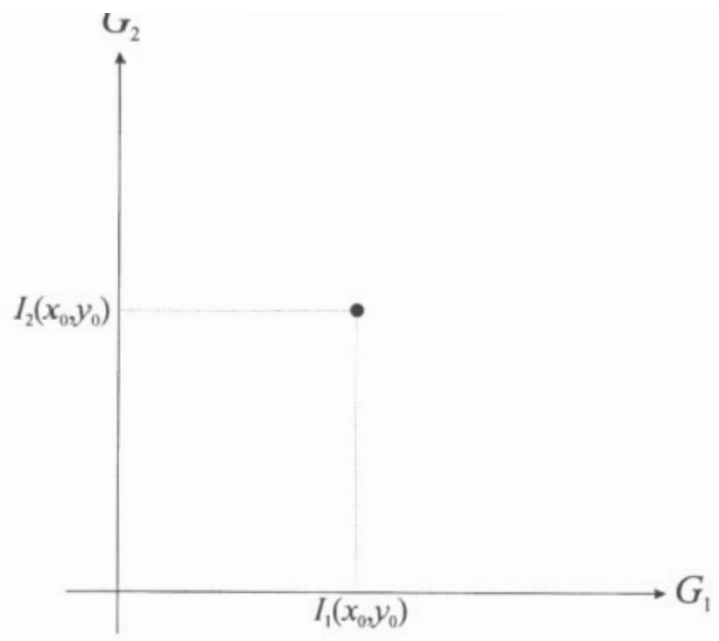

Figure 1. Representation of the correlation between the grey levels of images $I_{1}$ and $I_{2}$.

images, this transformation is point-dependent, that is for a given $G_{1}$ there will be in general several $G_{2}$.

Let us denote by $F$ the ideal functional relationship between $I_{1}$ and $I_{2}$, so that,

$$
I_{2}(x, y)=F\left(I_{1}(x, y)\right)
$$

so that, if we have an output grey level $G_{1}$ and a corresponding grey level $G_{2}$, then we will have, independently of the point where they have been measured,

$$
G_{2}=F\left(G_{1}\right) \text {. }
$$

If we substitute equation (4) in equations (2) and (3), we obtain

$$
F(T(E))=T(f(E)) .
$$

If we can determine $F$ and $f$ we can solve this equation, obtaining $T$, namely the irradiance response of the image-processing system. The dependence described by $f$ can be imposed, for example, by using a neutral filter of known optical density, so that $E_{1}$ and $E_{2}$ are related by

$$
E_{2}(x, y)=A E_{1}(x, y)
$$

where $A$ is the transmission coefficient of the filter. On the other hand, an estimation $F^{*}$ of $F$ can be obtained from the data $I_{1}(x, y)$ and $I_{2}(x, y)$ by adjusting them to some function by means of any standard technique of data fitting. We will show in section 4 how this estimation has been made in our case.

This supposes that, from (6), we only obtain an estimation of $T, T^{*}$, that fulfils

$$
F^{*}\left(T^{*}(E)\right)=T^{*}(f(E)) .
$$

Furthermore, this determination is only relative, because in (6), $E$ is a dummy variable, so the units of irradiance are lost in the process. To make possible an absolute estimation of $T$, only one value of the response for a given incident irradiance must be known.

\section{The experimental set-up}

To produce the images $E_{1}$ and $E_{2}$ we have used a $\mathrm{He}-\mathrm{Ne}$ laser beam. The only requirement for these images is that they produce the desired range of grey levels. We used simply two defocused images of the laser beam. Of course, the more equalized the histogram of the digitized image, the better for our purposes, but this is not a major constraint for the system. The use of monochromatic light makes easier the treatment of the results and covers almost all the practical situations in which our technique is useful. When white light is used, it is required that we know in a very accurate way its spectrum and also the spectral responsivity of the CCD camera. Therefore, all the results of this work are valid only for a wavelength of $632.8 \mathrm{~nm}$. Other wavelengths will produce a response curve essentially of the same shape, but multiplied by a factor depending on the gain due to the spectral responsivity of the camera and the interference effects produced in the several interfaces of the $\mathrm{CCD}$ detector. [4]

The experimental set-up is shown in figure 2. The laser beam is incident on a rotating diffuser, and then is collimated by a positive lens and projected on a screen. A CCD Sony CMA-D5CA camera records the image of the screen, $E_{1}$. A neutral filter can be placed in front of the objective lens of the CCD camera to obtain the second attenuated image $E_{2}$. These images were digitized by means of a Matrox MVP AT/NP processing board. We used two Melles-Griot fused silica neutral filters, whose optical densities were 0.1 and 0.03 (that is, transmittances of 0.794 and 0.933 respectively). There exist two natural positions for these filters in the set-up, denoted by $F 1$ and $F 2$ in figure 2 . We have used $F 2$ for reasons that will be discussed in section 4 . The use of the rotating diffuser breaks the spatial coherence of the beam, in order to avoid speckle and to produce smooth images. To reduce electronic noise due to the detectors, 50 images were averaged for each measurement.

To test the results obtained with our technique, we measured the response of the image-processing system (CCD plus board) with the arrangement shown in figure 3 . The laser beam power can be varied by the rotation of a polarizer $P$ placed between the laser output and the rotating diffuser $D$. The light coming from the diffuser is expanded by a positive lens and is incident on the stop $S$. The stop is placed far enough from the lens

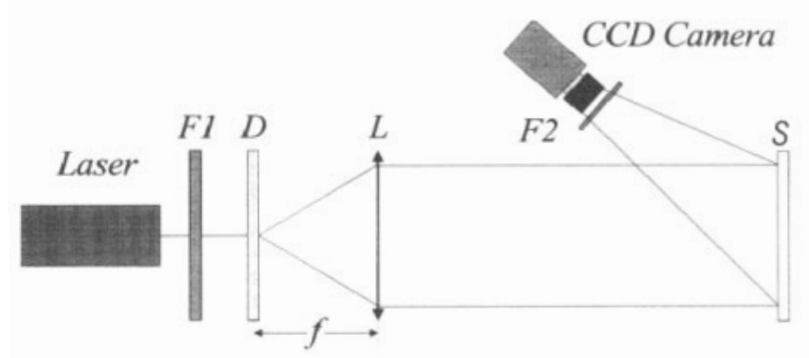

Figure 2. The experimental set-up for acquiring images. 


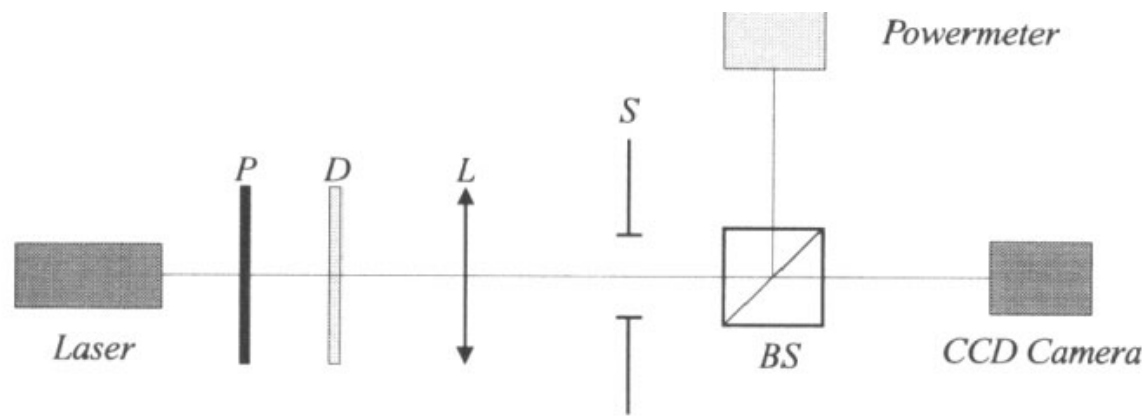

igure 3. The experimental set-up for the measurement of irradiance response.

(about $1 \mathrm{~m}$ ) to ensure that the light beam incident on it is of constant intensity over the whole aperture. Then the beam is divided by a non-polarizing $50: 50$ beamsplitter, BS. A powermeter and the CCD are placed symmetrically with respect to the beam-splitter. The non-polarizing beam-splitter and the symmetrical position guarantee that the power incident on the power meter and the CCD array are the same. The objective lens and anti-reflecting window of the camera were removed, so only the last protection film on the CCD detector array remained.

A remaining problem is that this protection film produces an interference fringe pattern on the image. This happens because, although the rotating diffuser breaks the spatial coherence of the beam, there still exists temporal coherence. The grey level produced by the image-processing system for each incident power was calculated by spatially averaging over the whole acquired image $(512 \times 512$ pixels). This procedure makes the measurement insensitive to the spatial fluctuations in grey level due to the interference fringe pattern. Of course, this may hide spurious fluctuations of the image irradiance. Then, care must be taken to assure that the image irradiance is uniform over the whole CCD detector. In our case, the method for producing the images ensures that.

All the measurements were taken after the system had warmed up (about $1 \mathrm{~h}$ ). The CCD camera offers two possibilities for gamma correction [4] denoted by gamma on and gamma off, which correspond to $\gamma=0.45$ and $\gamma=1$, so two sets of measurements were made. The results are shown in figure 4.

\section{Processing algorithms}

In this section we will discuss the algorithms used to solve equation ( 8 ) from the experimental measurements $I_{1}$ and $I_{2}$, defined by equations (2) and (3), and obtained with the arrangement depicted in section 3.

As we have said, we have used a neutral filter to obtain one of the images so $E_{1}$ and $E_{2}$ fulfil a relationship like the one given by equation (7), that is

$$
f(E)=A E \text {. }
$$

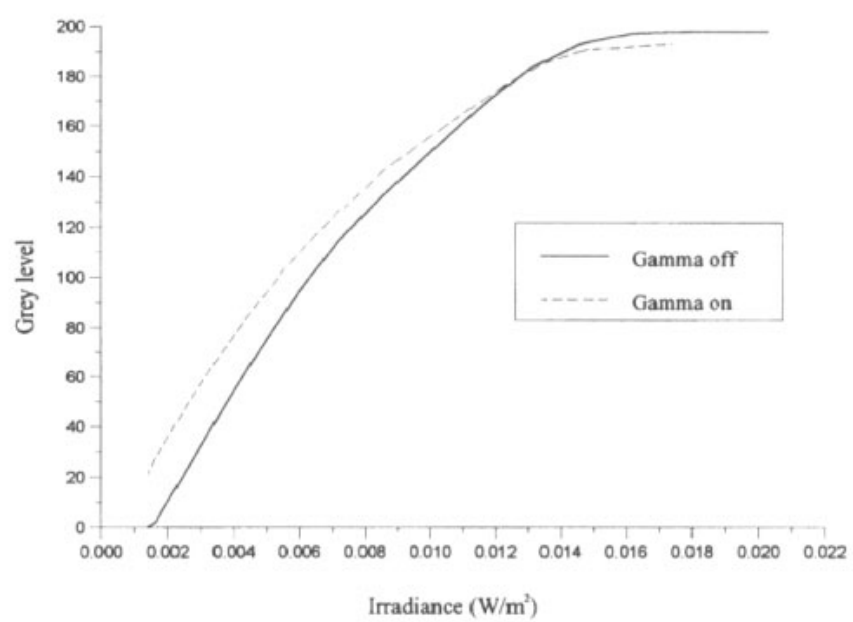

Figure 4. Measured irradiance responess for the chargecoupled device camera plus image processing board. The full line corresponds to the 'gamma off' option of the camera $(\gamma=1)$ and broken line to the 'Gamma on' option $(\gamma=0.45)$.

An example of the relationship between the two responses $I_{1}$ and $I_{2}$ is shown in figure 5 . As can be seen in figure 5 and as we have said in section 2 , there exists a dispersion in the data so a wide line is obtained instead of a thin one. From these data we must extract an estimation of the functional dependency $F^{*}$. To do this we could fit the data globally to a polynomical model of given degree. To make this process statistically reliable we must proceed step by step, adding terms of higher degree to the polynomical model until a reliability level is reached. In this way we would obtain an analytical function $F^{*}(x)=\Sigma n a_{n} x^{n}$, which can be introduced in equation (8). This method, however, presents some difficulties. It is not easy to program it and the reliability level must be chosen by the user. Also, the election of a polynomical model is one of many possibilities. To make the process independent of these arbitrary decisions we have proceeded in the following way.

(i) We construct a two-dimensional histogram of grey levels for $I_{1}$ and $I_{2}$, that is a matrix $\mathbf{H}$ with $H_{j, k}$ being the number of pixels in which a grey level of value $G_{1}=j$ of $I_{1}$ is transformed into a grey level $G_{2}=k$ of $I_{2}$, 


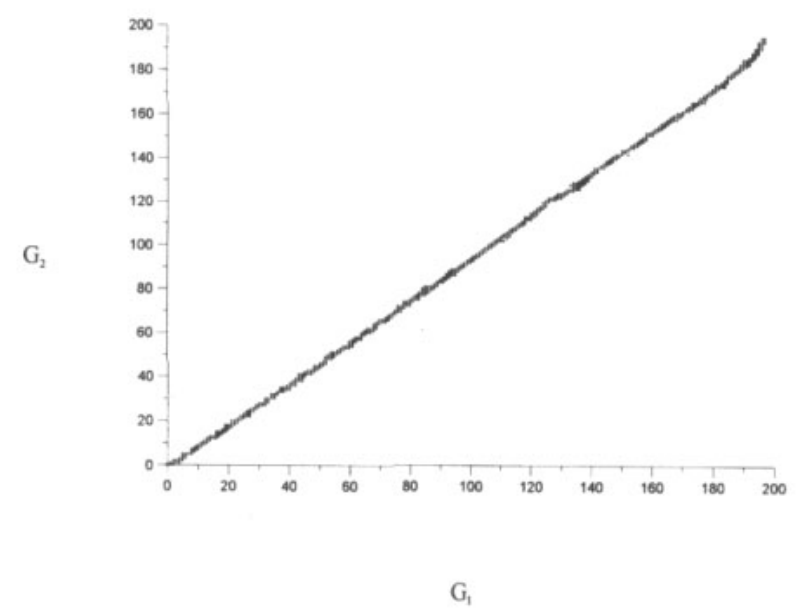

Figure 5. An example of the relationship between the grey levels of images $I_{1}$ and $I_{2}$. The widening of the line is produced by problems such as electronic noise, non-uniformities of the filters and misalignments.

with

$$
j=0,1 \ldots G_{2, \max } \quad k=0,1 \ldots G_{1, \max }
$$

where $G_{1, \max }$ and $G_{2, \max }$ are the maximum grey levels for $I_{1}$ and $I_{2}$ respectively. Obviously matrix $\mathbf{H}$ fulfils the criterion that

$$
\sum_{j, k} H_{j, k}=\text { total number of pixels on an image. }
$$

The matrix $\mathbf{H}$ corresponding to the data of figure 5 is represented in a three-dimensional image in figure 6.

(ii) Each column of matrix $\mathbf{H}$ corresponds to the distribution of grey levels $G_{2}$ for each grey level $G_{1}$. We calculate the first moment of each of these distributions forming an array $F^{*}$ kiven by

$$
F_{k}^{*}=\frac{\sum_{j=0}^{G_{2, \max }} j H_{j, k}}{\sum_{j=0}^{G_{2, \max }} H_{j, k}} \quad k=0,1 \ldots G_{1, \max } .
$$

The array $F^{*}$ corresponding to the matrix $\mathbf{H}$ of figure 6 is shown in figure 7 .

(iii) The array calculated in step (ii) is used as a sampled form of the estimator $F^{*}$.

Once we have obtained by steps (i)-(iii) the sampled estimation $F^{*}$, whose elements are given by equation (12), we need to solve equation (8). If we introduce (9) in (8) we will obtain

$$
F^{*}\left(T^{*}(E)\right)=T^{*}(A E)
$$

Equation (13) relates the values of $T^{*}$ for two different arguments, namely $A E$ and $E$.

To solve analytically equation (13) is at least difficult. For this reason we have solved it by a numerical procedure, obtaining a vectorial solution for $T^{*}$ given by pairs $\left(E_{n}, T^{*}{ }_{n}\right)$. For the starting point $T^{*}{ }_{0}$ we chose an arbitrary value in the dynamical range of grey levels of the images $I_{1}$ and $I_{2}$. For instance, if we know that

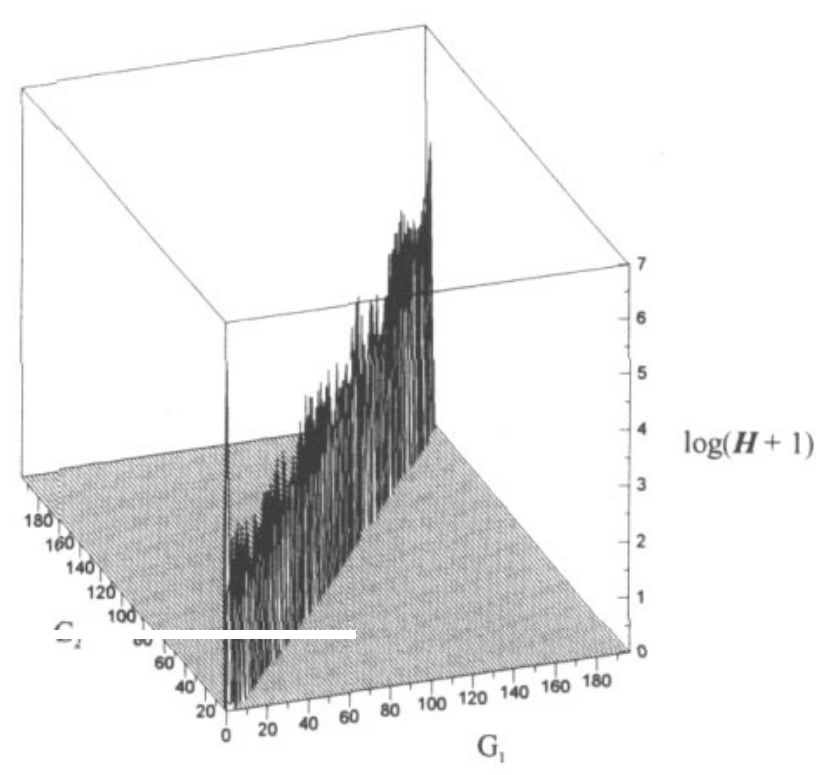

Figure 6. A three-dimensional representation of matrix $\mathbf{H}$ corresponding to the data of figure 5 . For display purposes, inniH +11 is renresentert insteari af $H$

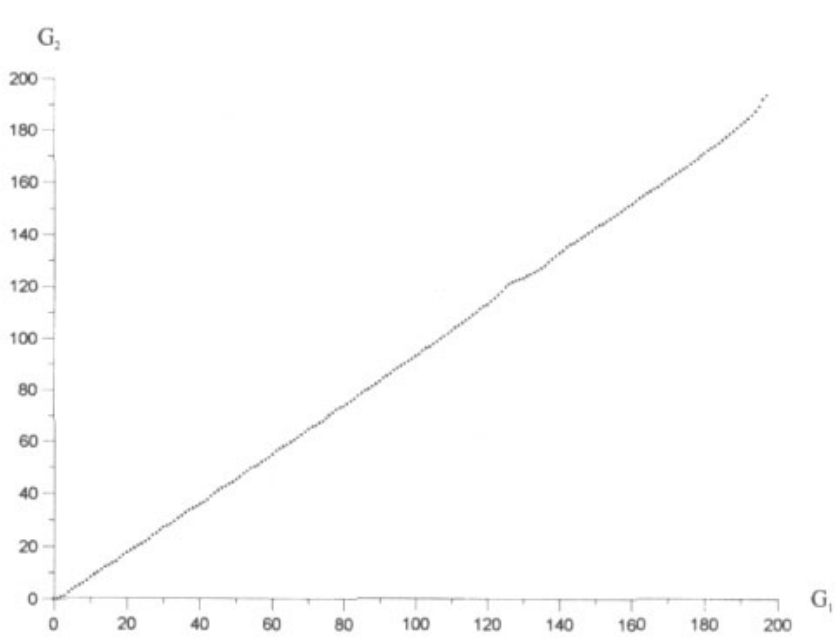

Figure 7. The discrete estimation for $F, F^{*}$, for the data of figure 5.

the saturation of the system corresponds to a grey level of 180 , then we can choose a grey level of 150 to start the algorithm.

With this initial value we can calculate all the other values of $T^{*}$ by the following equations:

$$
\begin{array}{cc}
T_{1}^{*}=F^{*}\left(T_{0}^{*}\right)=T^{*}\left(A E_{0}\right) & E_{1}=A E_{0} \\
T_{2}^{*}=F^{*}\left(T_{1}^{*}\right)=T^{*}\left(A E_{1}\right) & E_{2}=A E_{1} \\
T_{n}^{*}=F^{*}\left(T_{n-1}^{*}\right)=T^{*}\left(A E_{n-1}\right) & E_{n}=A E_{n-1}
\end{array}
$$

In each step, $T_{n}^{*}$ is calculated from the value of $T_{n-1}^{*}$ using the table $F_{k}^{*}$ of sampled values of $F^{*}$. Since $F_{k}^{*}$ and therefore $T_{n}^{*}$ may be non-integer, sometimes we will need to calculate $F^{*}$ for a non-integer argument. In 
that case, as can be seen in figure 8, we make an interpolation using the neighbourhood of the point. For practical purposes we have observed that a linear interpolation with three neighbours is enough.

As equation (13) shows, the iterative calculations for $T_{n}^{*}$ and $E_{n}$ are independent. That means that $E_{0}$ is a free parameter, or, in other words, that the solution offered by equations (13) is a relative one unless we know one absolute value for the response $\left(T_{a}^{*}, E_{a}\right)$, which can be used as starting point to generate the absolute response.

In (13) $A<1$; this means that the pairs of the response curve are calculated towards zero. We need to complete the process described in (13) for values bigger than $E_{0}$ in order to obtain the function $T^{*}$ for the whole range of possible grey levels. To do this, we would need to use (13) with $A>1$. We can achieve this by repeating the steps (i)-(iii) used to calculate $F_{k}^{*}$ but with the transposed matrix $\mathbf{H}$ instead of $\mathbf{H}$. That is, considering as first image $I_{2}$ and as second image $I_{1}$.

In the way explained above, we can obtain the relative irradiance response of the system. If we are interested in an absolute determination of this response then we must use, as we have said, an initial value $E_{a}$ for which $T\left(E_{a}\right)$ is known. Since $E_{a}$ and $T\left(E_{a}\right)$ are affected by a certain amount of experimental error, this error will propagate through the recursive process of calculation. This implies an amplifying of this error for $A>1$, and a diminishing of errors for $A<1$. Therefore, we must choose a high value for the starting point $E_{a}$ to avoid as much as possible the ascending iterations. This process of propagation of error can be seen in figure 9.

The choice of the value $A$ and its experimental implementation (the placement and tolerances of the actual filters) are practical problems that must be taken into account.

First, there must exist a compromise for the value $A$. If $A$ is near to 1 , then the algorithm described by (13) will produce a very well sampled $T^{*}$, but for low grey levels the difference between the two images $I_{1}$ and

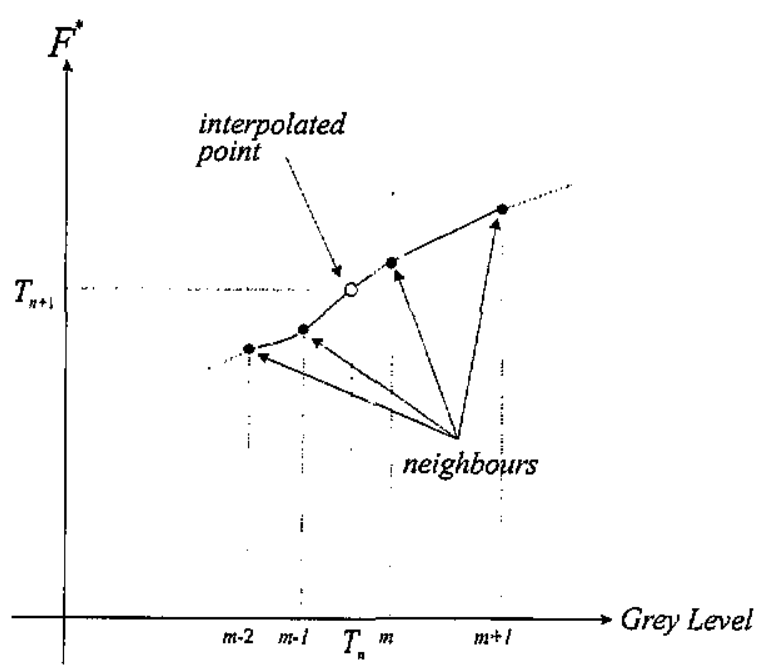

Figure 8. A schematic representation of the interpolation process.
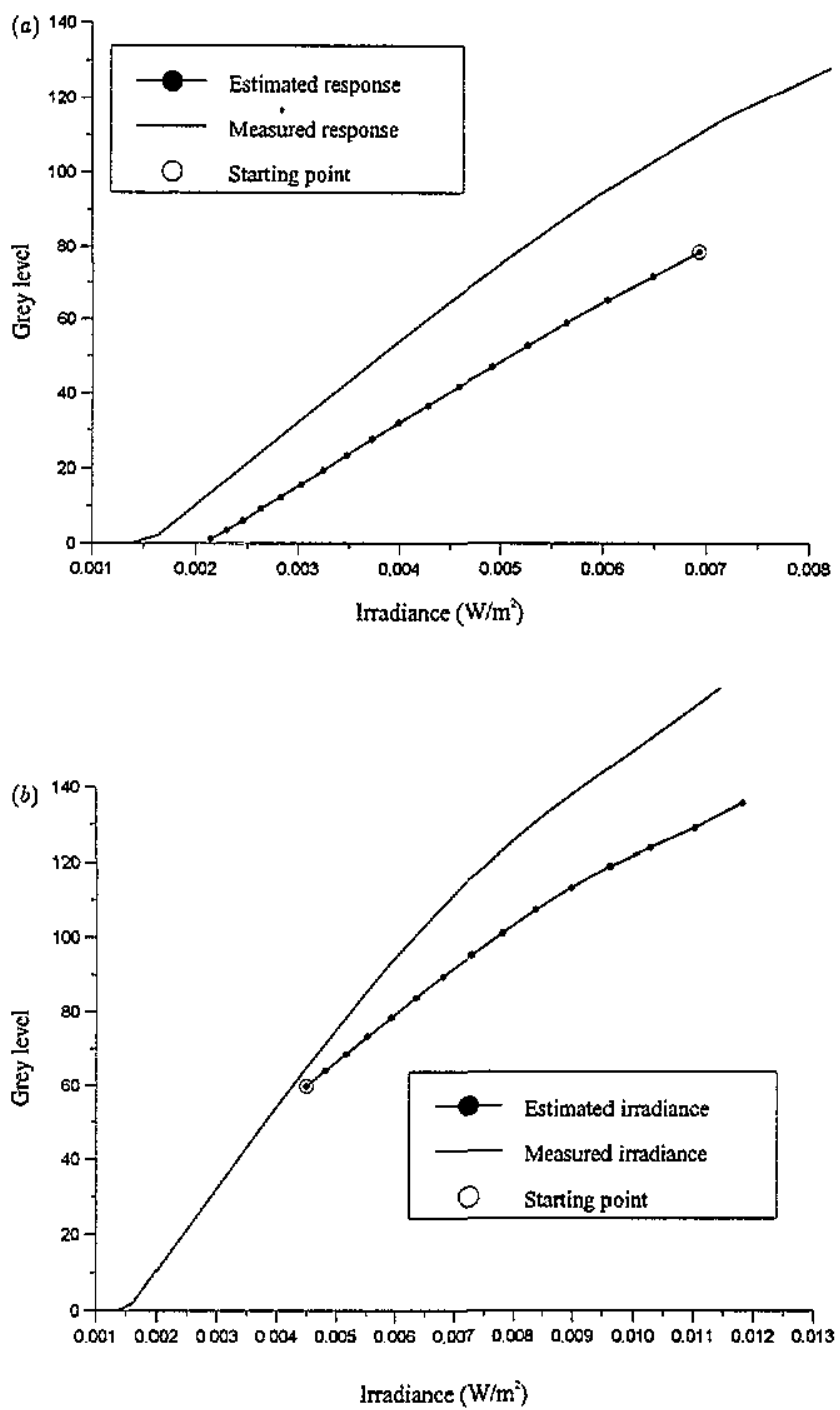

Figure 9. Propagation of errors in the algorithm when $A<1$ (a) and when $A>1$ (b). The full line corresponds to the measured irradiance and the dotted line to the estimated irradiance. The starting point of the algorithm is denoted by an open circle.

$I_{2}$ is almost inexistent, so the correlation between them provides little information. On the other hand, if $A$ is too small (say, 0.5 ) then we will be able to extract much information from the correlation, but $T^{*}$ will be poorly sampled. This is shown in figure 10 . Two values for $A$ have been used, $A_{1}=0.933$ and $A_{2}=0.794$. The adjustment for $A_{1}$ (figures $10(b)$ and $(d)$ ) is clearly worse than for $A_{2}$ (figures $10(a)$ and $(c)$ ), especially for low grey levels, so a good compromise value is $A=0.794$.

Second, the placement of the filter can influence the measurements. A filter may present spatial fluctuations in its transmittance value $A$. These fluctuations act as another source of errors in the measurements. The consequences of this are very different depending on the extent of the illuminated area of the filter. If it is very small (position $F_{1}$ of figure 2 , non-expanded laser beam), then the actual value of $A$ for this illuminated zone will differ from the nominal value of $A$, and therefore the algorithm (13) will produce false results when a nominal value of $A$ is used in the equations. We have observed 

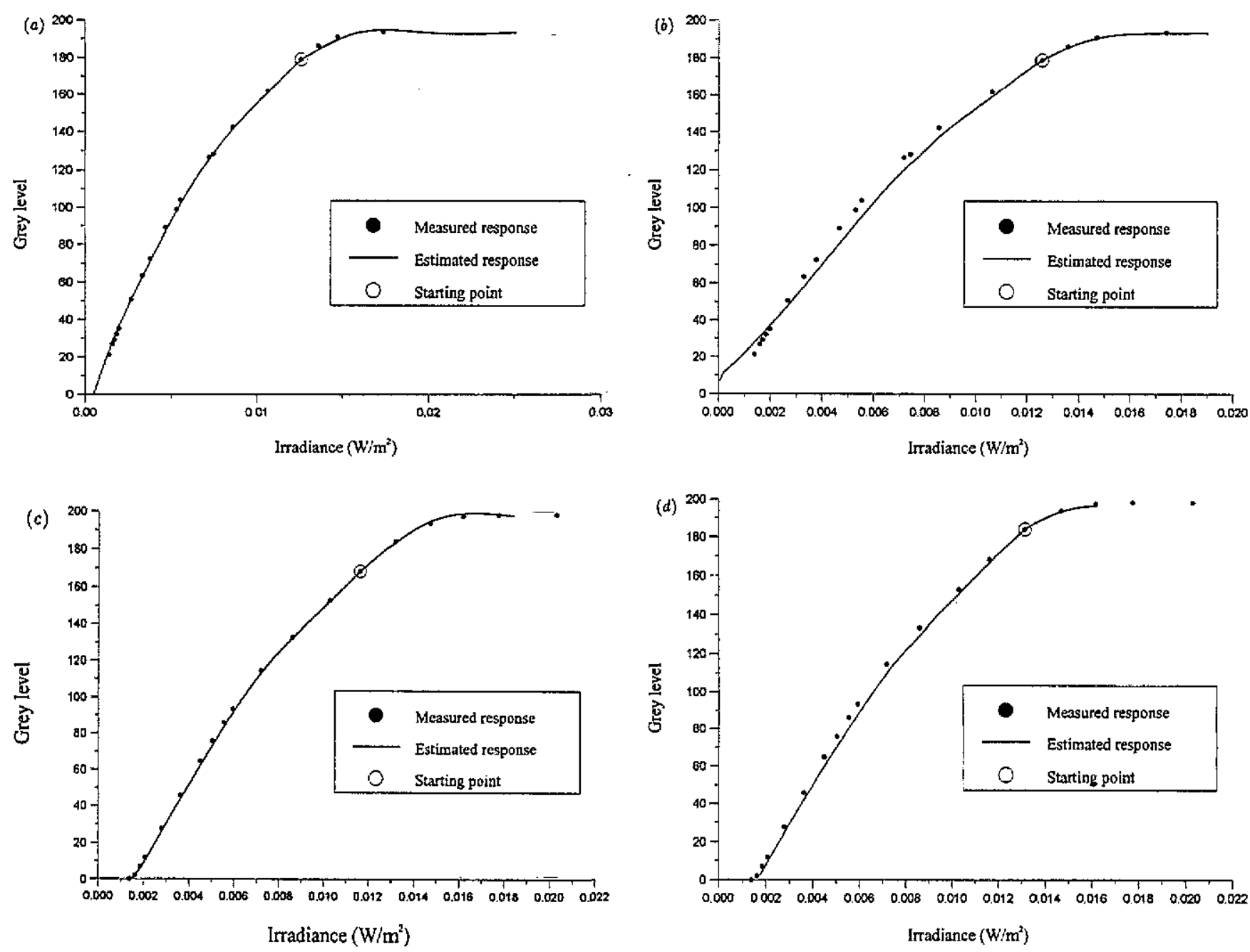

Figure 10. A comparison between the measured and the estimated response of the image processing system for (a) gamma on, $A=$ 0.794; (b) gamma on, $A=0.933$; (c) gamma off, $A=0.794$; and ( $d$ ) gamma off, $A=0.933$. The full lines correspond to the estimated response, full circles to the measured points and open circles to the starting points of the algorithm.

that, for $A_{1}$, an error in transmittance of $1 \%$ is not tolerable for the algorithm. However, this error is acceptable for $A_{2}$. This is due to the fact that the information about $T^{*}$ in the correlation for $A_{2}$ is greater than that for $A_{1}$.

On the other hand, if we place the filter in position $F_{2}$ then we are using almost all the area of the filter, and the above-mentioned fluctuations will not produce an error in the nominal value $A$ (since it is averaged over the whole area) but rather a widening of the correlation curve (figure 5), which produces a small degradation of the correlation. For these reasons, we have chosen position $F_{2}$ for the placement of the filters.

Finally, lateral shifts of the image introduced by misalignment of the filter must be avoided. These lateral shifts can produce an appreciable decorrelation between the images, and a subsequential widening of the correlation curve.

\section{Experimental results}

We have measured using the depicted technique the irradiance response of the image-processing system for two different stops and for two different values of gamma correction. In all the cases we have used as starting point an irradiance for which the response of the system is known, in order to prove that curves for the absolute response of the system can be obtained and coincide with the real ones (figure 4). We do not need to do this if we are only interested in knowing the nonlinearities of the response. The results are shown in figure 10. As can be seen and as we have commented in section 4 , the agreement between the measured response and the response obtained by our technique for $A=0.794$ (figures $10(a)$ and $(c))$ is very good, while for $A=0.933$ (figures $10(b)$ and $(d)$ ) the results are worse.

These results have been achieved by implementing the algorithm in a PC 486 at $66 \mathrm{MHz}$ by using a MATLAB program. Once the images have been digitized and stored in the computer bard disc, the processing time is typically of the order of $3 \mathrm{~s}$.

\section{Conclusions}

We have presented a method that permits one to estimate in a fast and simple way the irradiance response of an 
image-processing system. The experimental set-up and the algorithms employed are very easy to implement. The agreement between theory and experiment is very good. This method can be used to monitor the behaviour of the system as it changes, for instance, when it warms up.

\section{Acknowledgment}

This work was partially supported by a PETRI (Programa de Estímulo a la Tranferencia de Resultados de Investigación) grant, project PTR 93-0092.

\section{References}

[1] Ruff J A and Siegman A E 1992 Single-pulse laser beam quality measurements using a CCD camera system $\mathrm{Appl}$. Opt. 31 4907-9

[2] Creath K 1988 Phase-measurement interferometry techniques in Progress in Optics XXVI pp 349-93

[3] Rea M S and Jeffrey I G $1990 \mathrm{~A}$ new luminance and image analysis system for lighting and vision I. Equipment and calibration J. Mlluminating Eng. Soc. winter 64-72

[4] Spooren R 1994 Standard charge-coupled device cameras for video speckle interferometry Opt. Eng. 33 889-96 